\title{
Erratum: Persistence-Driven Durotaxis: Generic, Directed Motility in Rigidity Gradients [Phys. Rev. Lett. 118, 078103 (2017)]
}

\author{
Elizaveta A. Novikova, Matthew Raab, Dennis E. Discher, and Cornelis Storm
}

(2) (Received 11 September 2018; published 11 October 2018)

DOI: 10.1103/PhysRevLett.121.159901

In deriving continuum equation (5) for the probability distribution $\mathrm{P}(x, t)=\rho_{+}(x, t)+\rho_{-}(x, t)$ from Eqs. (3) and (4) for the densities of left and right movers, we overlooked a term proportional to the difference between $\rho_{-}$and $\rho_{+}$. As a result, Eq. (5) is incorrect. In the case that the cell velocity $v_{c}$ is uniformly constant, it should read

$$
\partial_{t}^{2} \mathrm{P}+2 \lambda(x) \partial_{t} \mathrm{P}=v_{c}^{2} \partial_{x}^{2} \mathrm{P}-2 v_{c}\left[\partial_{x} \lambda(x)\right]\left(\rho_{-}-\rho_{+}\right) .
$$

As this equation cannot be transformed into a single, closed equation involving only the probability density $\mathrm{P}(x, t)$ we suggest that, in the case of a uniformly constant cell velocity $v_{c}$, the coupled equations for the left- and right-moving densities are a convenient starting point for continuum analysis of persistence-driven durotaxis

$$
\begin{aligned}
& \partial_{t} \rho_{+}=-v_{c} \partial_{x} \rho_{+}+\lambda(x)\left(\rho_{-}-\rho_{+}\right), \\
& \partial_{t} \rho_{-}=v_{c} \partial_{x} \rho_{-}-\lambda(x)\left(\rho_{-}-\rho_{+}\right) .
\end{aligned}
$$

The same error that led to our omission of the last term on the right-hand side of Eq. (E1) led us to erroneously state, directly below Eq. (5), that a position-dependent velocity could be included by simply replacing $v_{c} \rightarrow v_{c}(x)$ in the original Eq. (5). The correct way to treat a position-dependent velocity and an analysis of the resulting transport are detailed in Ref. [1].

The central conclusions of our Letter are unaffected by the above error; direct numerical integration of Eqs. (E2) confirms their solutions agree with numerical results for the discrete walker model on which our main conclusions are basedsee Fig. 1.

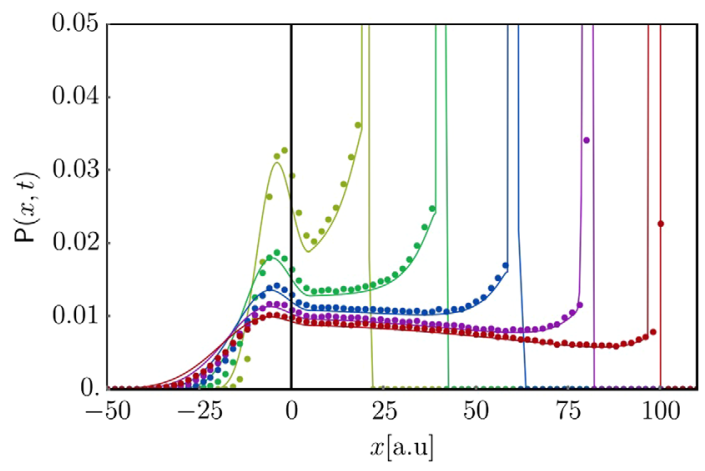

FIG. 1. Comparison between discrete simulation and numerical solutions to Eqs. (E2). Probability distributions $\mathrm{P}(x, t)$ determined by direct integration of Eq. (E2), subject to the initial conditions $\rho_{+}(x, 0)=\rho_{-}(x, 0)=\frac{1}{2} \delta(x)$ (solid lines) and that same distribution measured in a discrete random walker simulation (dots). In both cases, the turning frequency $\lambda(x)$ decreased linearly from 0.5 to 0.02 over the $x$ interval $[-5,5]$, and $v_{c}=1$. From left to right, we plot distributions for $t=20 \ldots 100$ at 20 time unit intervals. 
We are grateful to professors Charles R. Doering, Xiaoming Mao, and Leonard M. Sander for bringing this error to our attention.

[1] C. R. Doering, X. Mao, and L. M. Sander, Phys. Biol. 15, 066009 (2018). 